\title{
NONEXTENSIVE STATISTICAL MECHANICS APPLICATION TO VIBRATIONAL DYNAMICS OF PROTEIN FOLDING
}

\begin{abstract}
Ethem Aktürk* and Handan Arkınt
Department of Physics Engineering, Hacettepe University, 06800 Ankara, Turkey

The vibrational dynamics of protein folding is analyzed in the framework of Tsallis thermostatistics. The generalized partition functions, internal energies, free energies and temperature factor (or Debye-Waller factor) are calculated. It has also been observed that the temperature factor is dependent on the non-extensive parameter q which behaves like a scale parameter in the harmonic oscillator model. As $q \rightarrow 1$, we also show that these approximations agree with the result of Gaussian network model.
\end{abstract}

Keywords: Tsallis thermostatistics, Harmonic Oscillator, Protein Folding, Temperature Factor

PACS numbers: 05.20.-y,87.10.te,05.70.-a

\section{INTRODUCTION}

The statistical mechanics paradigm based on Boltzmann-Gibbs entropy which is great success looks after be incapable to deal with many interesting physical systems[1]. In 1988, Tsallis advanced a nonextensive generalization of Boltzamann-Gibbs entropic measure. There are many applications for Tsallis formalism such as the specific heat of the harmonic oscillator [2], one-dimensional Ising model [3], the Boltzmann H-theorem [4], the Ehrenfest theorem [5], quantum statistics [6], paramagnetic systems [7], Cercle maps [8], Henon map [9], Haldane exclusion statistics [10], q-expectation value[11], quantum mechanical treatment of constraints[12]. In this paper, we will focus on thermodynamics and

*E-mail: eakturk@hacettepe.edu.tr

†E-mail: handan@hacettepe.edu.tr 
vibration properties of proteins in the framework of a generalized thermostatistics. Before proceeding with generalized gaussian network model, we described fundamental definitions of Tsallis thermostatistics in Section II. We then study generalized gaussian network model and result in section III. Results will be discussed in section IV.

\section{FUNDAMENTAL DEFINITIONS OF TSALLIS THERMOSTATISTICS}

The generalization which has been successfully nonextensive statistical mechanics is based on the following expression [13]

$$
S_{q}=k \frac{1-\operatorname{Tr}\left(\rho^{q}\right)}{q-1} .
$$

In the Tsallis statistics, the nonextensive canonical distribution is given by

$$
\rho_{q}=\frac{1}{Z_{q}}[1-(1-q) \beta H]^{1 /(1-q)},
$$

where

$$
Z_{q}=\int \prod_{n=1}^{N} d p_{n} d x_{n}[1-(1-q) \beta H]^{1 /(1-q)},
$$

is the partition function with $q \in \mathbf{R}$ and $\beta \equiv 1 / T$ (with $k=1$ ). Eq.(2) is obtained maximizing the Tsallis entropy using lagrange multiply methods [13, 14]. The internal energy is described by

$$
U_{q}=\int \prod_{n=1}^{N} d p_{n} d x_{n} \rho^{q} H
$$

where

$$
\int \prod_{n=1}^{N} \mathrm{~d} p_{n} \mathrm{~d} x_{n} \rho^{q}=1
$$

In Free energy notation, Eq.(4) appears as

$$
U_{q}=F_{q}-T \partial F_{q} / \partial T,
$$

where $F_{q}$ is free energy is described as

$$
F_{q}=\left(Z_{q}^{q-1}-1\right) /[(1-q) \beta] .
$$

The expectation of an observable $\mathrm{A}$ is

$$
A_{q}=\langle A\rangle_{q}=\int \prod_{n=1}^{N} d p_{n} d x_{n} \rho^{q} .
$$




\section{GENERALIZED GAUSSIAN NETWORK MODEL AND RESULTS}

We focus our discussion on the classical Tsallis statistics by considering protein folding and harmonic approximation. Consider the following Hamiltonian for Gaussian Network Model(GNM) based on harmonic approximation[15]

$$
H=\sum_{i=1}^{N} \frac{p_{i}^{2}}{2 m}+\frac{\gamma}{2} \Delta R_{i}^{T} L \Delta R_{i}
$$

where the first term is the kinetic energy of the system and $\gamma$ is the strength of the springs. In this model, springs are assumed as homogeneous. $R_{i}$ and $\Delta R_{i}$ is also defined as the equilibrium position and the displacement with respect to $R_{i}$ of the $i$-th $\mathrm{C}_{\alpha}$ atoms. The model is eventually described by the contact matrix $L$ with entries: $L_{i j}=1$ if the distance $\left|\mathbf{R}_{i}-\mathbf{R}_{j}\right|$ between two $\mathrm{C}_{\alpha}$ 's, in the native conformation, is below the cutoff $R_{0}$, while is 0

otherwise. From Eq.(3), partition function for the Gaussian Network model[15] which has above hamiltonian is given by

$$
\begin{aligned}
Z_{q}= & \int \prod_{i=1}^{N} \mathrm{~d} p_{i} \mathrm{~d} \Delta R_{i}[1-(1-q) \beta \\
& \left.\times\left(\sum_{i=1}^{N} \frac{p_{i}^{2}}{2 m}+\frac{\gamma}{2} \Delta R_{i}^{T} L \Delta R_{i}\right)\right]^{1 /(1-q)} .
\end{aligned}
$$

we introduce the variables $L=V^{T} \lambda V, x=V \Delta R$ and thus $\Delta R^{T} L \Delta R=x^{T} \lambda x$ if $\lambda$ is eigenvalues of the $L$ and $V$ is eigenvectors of $L$. We can rewrite partition function as:

$$
\begin{aligned}
Z_{q}= & \int \prod_{i=1}^{N} \mathrm{~d} p_{i} \mathrm{~d} x_{i}[1-(1-q) \beta \\
& \left.\times\left(\sum_{i=1}^{N} \frac{p_{i}^{2}}{2 m}+\frac{\gamma}{2} \lambda_{i} x_{i}^{2}\right)\right]^{1 /(1-q)} .
\end{aligned}
$$

To calculate this integral, we definition new variables: $y_{i}=[(1-q) \gamma \lambda i \beta / 2]^{1 / 2} x_{i}$ and $y_{N+i}=$ $[(1-q) \beta /(2 m)]^{1 / 2} p_{i}[16]$, where $i=1,2,3, \ldots, N$. Substituting these variables into $Z_{q}$ gives

$$
\begin{aligned}
Z_{q}= & \left\{\prod_{i}^{N}\left[\frac{2}{(1-q) \lambda_{i} \beta}\left(\frac{m}{\gamma}\right)^{1 / 2}\right]\right\} \\
& \times \int \prod_{n=1}^{2 N} \mathrm{~d} y_{n}\left(1-\sum_{k=1}^{2 N} y_{k}^{2}\right)^{1 /(1-q)} .
\end{aligned}
$$


By using hyperspherical coordinates with $u=\left(\sum_{n=1}^{2 N} y_{n}^{2}\right)^{1 / 2}$ and calculating the integral over the angular variables[16], we obtain

$$
\begin{aligned}
Z_{q}= & \left\{\prod_{n=1}^{N}\left[\frac{2}{(1-q) \lambda_{n} \beta}\left(\frac{m}{\gamma}\right)^{1 / 2}\right]\right\} \frac{\Omega_{2 N}}{2} \\
& \times \int_{0}^{1} \mathrm{~d} u u^{N-1}(1-u)^{1 /(1-q)} .
\end{aligned}
$$

By using definition of the solid angle[17] $\Omega_{2 N}=2 \pi^{N} / \Gamma(N)$ and using integral representation of Euler beta function[18], we obtained that

$$
\begin{aligned}
Z_{q} & =\left\{\prod_{n=1}^{N}\left[\frac{2 \pi}{(1-q) \lambda_{n} \beta}\left(\frac{m}{\gamma}\right)^{1 / 2}\right]\right\} \frac{\Gamma\left(\frac{1}{1-q}+1\right)}{\Gamma\left(\frac{1}{1-q}+1+N\right)} \\
& =\left[\left(\frac{2-q}{1-q}\right)_{N}\right]^{-1} \prod_{n=1}^{N}\left[\frac{2 \pi}{(1-q) \lambda_{n} \beta}\left(\frac{m}{\gamma}\right)^{1 / 2}\right]
\end{aligned}
$$

where $(a)_{n}=a(a+1)(a+2) \ldots(a+n-1)=\frac{(a+n-1) !}{(a-1) !}$ is the Pochhammer symbol [18]. Free energy of the system is given by

$$
F_{q}=-\frac{1}{\beta} \ln _{q} Z_{q}
$$

and

$$
F_{q}=-\frac{1}{\beta} \ln _{q}\left[\left(\frac{2-q}{1-q}\right)_{N}\right]^{-1} \prod_{n=1}^{N}\left[\frac{2 \pi}{(1-q) \lambda_{n} \beta}\left(\frac{m}{\gamma}\right)^{1 / 2}\right]
$$

and then

$$
F_{q}=-\frac{1}{\beta} \ln _{q}\left[\left(\frac{2-q}{1-q}\right)_{N}\right]^{-1}\left[\frac{2 \pi}{(1-q) \beta}\left(\frac{m}{\gamma}\right)^{1 / 2}\right]^{N}\left(\operatorname{det}\left(L^{-1}\right)\right)^{1 / 2} .
$$

From Eq.(6), the generalized internal energy can be calculated as

$$
U_{q}=\frac{N}{\beta} Z_{q}^{1-q} \frac{N}{\beta}\left\{\left[\frac{2 \pi}{(1-q) \beta}\left(\frac{m}{\gamma}\right)^{1 / 2}\right]^{N}\left(\operatorname{det}\left(L^{-1}\right)\right)^{1 / 2}\left[\left(\frac{2-q}{1-q}\right)_{N}\right]^{-1}\right\}^{1-q} .
$$

Hence, we obtained thermodynamic properties of the generalized Gaussian network model. On the other hand, the most properties of Gaussian network model is called temperature factor. The crystallographic temperature factors or B-factors, which is defined as intrinsic fluctuations of the atoms in crystal, is directly related to this fluctuations. The X-ray 
crystallographic temperature factors are used by the mean square fluctuation of $\mathrm{C}_{\alpha}$ atoms around their native positions[15]. The generalized temperature factor is expressed as

$$
B_{i q}(T)=\frac{8 \pi^{2}}{3}\left\langle x_{i} x_{i}\right\rangle_{q}
$$

with $\langle\cdot\rangle$ indicating the thermal average. Where $\left\langle x_{i} x_{j}\right\rangle_{q}$ is defined by

$$
\left\langle x_{i} x_{j}\right\rangle_{q}=\frac{1}{Z_{q}^{q}} \int D x D p x_{i} x_{j}[1-(1-q) \beta H]^{\frac{q}{1-q}},
$$

or

$$
\left\langle x_{i} x_{j}\right\rangle_{q}=\frac{1}{Z_{q}^{q}} \int D x D p x_{i} x_{j}\left[1-(1-q) \beta \sum_{i=1}^{N} \frac{p_{i}^{2}}{2 m}+\frac{\gamma}{2} \lambda_{i} x_{i}^{2}\right]^{\frac{q}{1-q}} .
$$

In the GGNM (generalized gaussian network model) approximation, this average is easily carried out, because amounts to a nonGaussian integration, and B-factors can be expressed in terms of the diagonal part of the inverse of the matrix

$$
\left\langle x_{i} \cdot x_{j}\right\rangle_{q}=\frac{3 k_{B} T}{\gamma}\left[\frac{L_{i j}^{-1}}{1+(1-q)(N+1)}\right]
$$

Substituting Eq. 20 into Eq.17 gives

$$
B_{i q}(T)=\frac{8 \pi^{2} k_{B} T}{\gamma}\left[\frac{L_{i i}^{-1}}{1+(1-q)(N+1)}\right] .
$$

If $q \rightarrow 1$, it is calculated that same result of the gaussian network model as[19]

$$
B_{i}(T)=\frac{8 \pi^{2} k_{B} T}{\gamma}\left[L_{i i}^{-1}\right]
$$

For the numerical results, we choice a reasonable cutoff distance including all residues pairs within a first interaction shell is $7.0 \AA$. We display in Fig 1 temperature factors for T4 lysozyme (or 3LZM pdb) as a function of residue index at $q=0.99$. Bold and dashed curves depict the experimental and theoretical results, respectively. The theoretical curve is normalized by taking $\gamma\left(\AA^{-2}\right)=2.06 k_{B} T$, therefore as to match the area enclosed by the two curves. The agreement between the theoretical and experimental curves is remarkable. In Fig,2, we also display to behavior cross correlation between i.residues and j.residues of all modes at $\mathrm{q}=0.99$. 


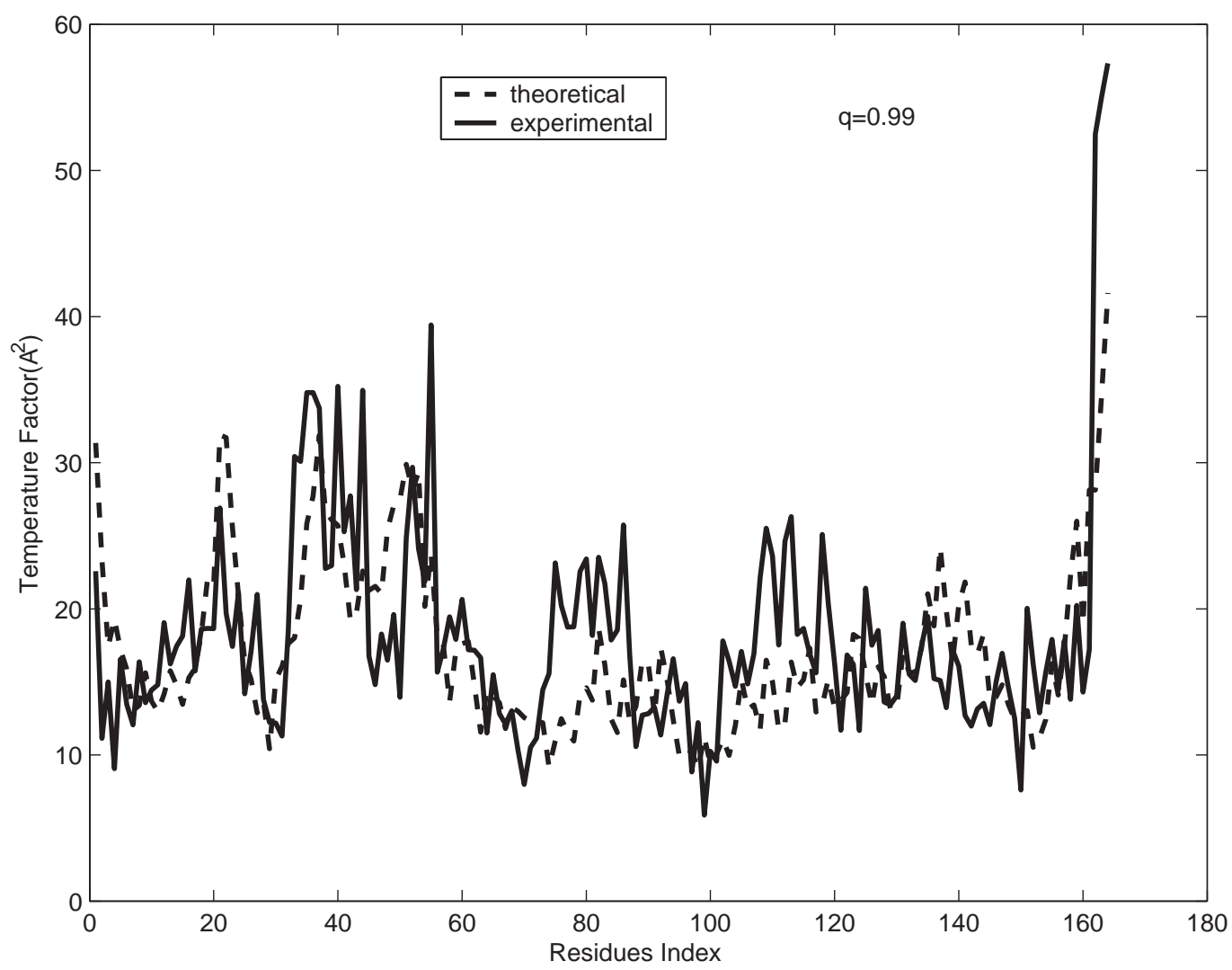

FIG. 1: Temperature factors for T4 lysozyme

\section{CONCLUSION}

In this study, we obtained temperature factor or Beta factor using by Tsallis statistical mechanics. In this framework, by the approximate scheme which we have used here, closed and analytical expressions for the generalized gaussian network model have been derived for harmonic oscillator. In addition to this, the temperature factor is q dependent. Since the temperature factor must be positive, $q$ must be less than unity. This also shows that $q$ must be less than unity for harmonic oscillator system. As can be seen in Fig 1, at q=0.99, the beta factors which are obtained from experimental and numerical calculation are in agreement with each other. The more the q parameter decreases, the more the temperature factor disagrees with experimental data. Therefore, for $\mathrm{q}$ values in the lower vicinity of 1, the experimental data are in agreement with numerical calculations. In this system, as $q \rightarrow 1$, it is observed that, both of the above expressions reduce to the results obtained in the standard Boltzmann-Gibbs statistics. 


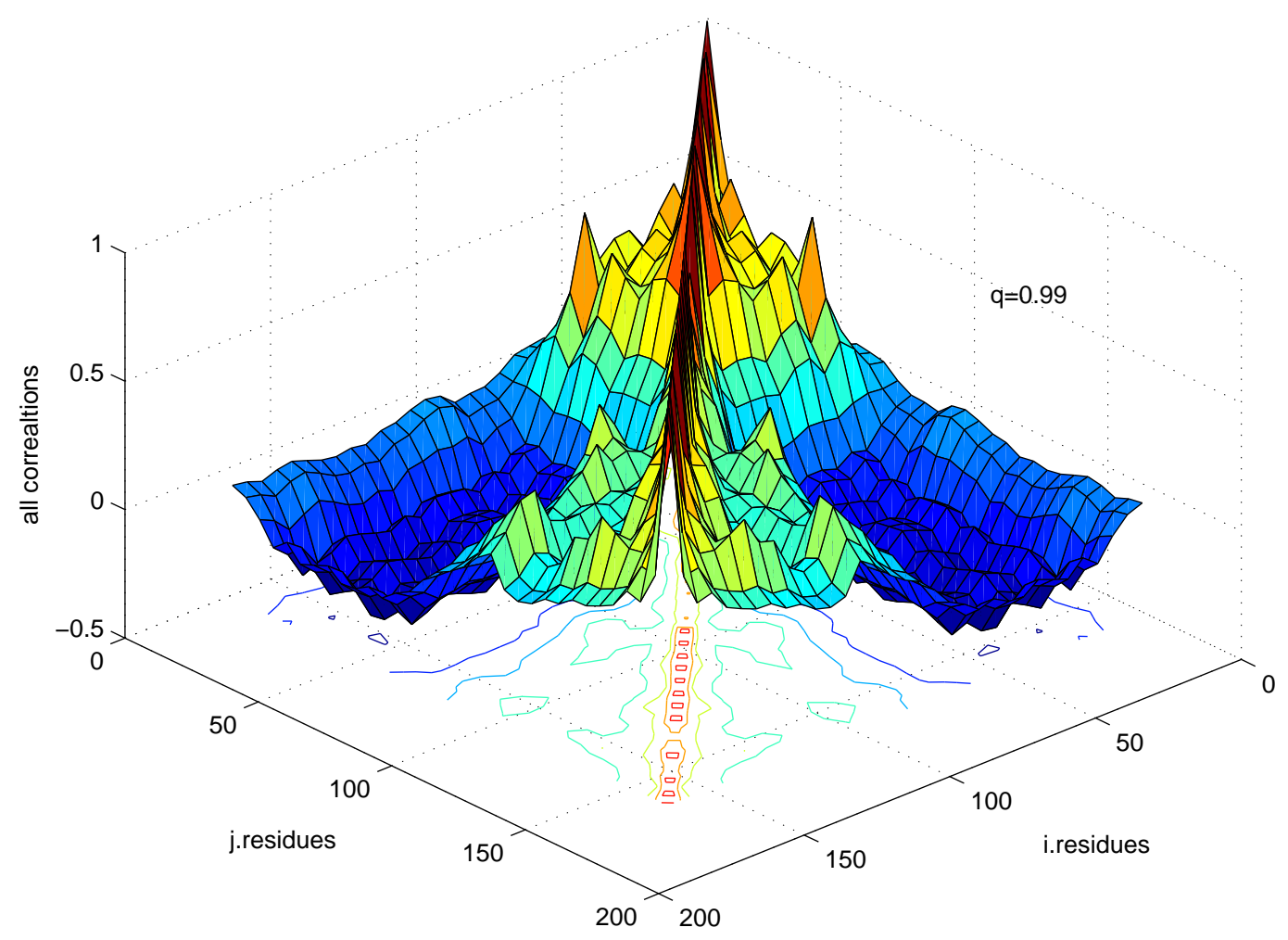

FIG. 2: Cross correlation between pair residues for T4 lysozyme

\section{ACKNOWLEDGEMENTS}

H.A. acknowledges support by the TÜBİTAK under the project number $104 \mathrm{~T} 150$ and from TÜBA under the Programme to Reward Successful Young Scientists.

[1] C. Tsallis, R. S. Mendes and A. R. Plastino, Physica A 261, 534 (1998).

[2] N. Ito and C. Tsallis, Nuovo Cimento 11,907 (1989).

[3] R.F.S. Andrade, Physica A 175, 285 (1991); A 203, 486 (1994).

[4] A.M. Mariz, Phys. Lett. A 165, 409 (1992); J.D. Ramshaw, Phys. Lett. A 175, 169 and 171 (1993).

[5] A. Plastino and A.R. Plastino, Phys. Lett. A 177, 177 (1993).

[6] F. Büyükkılıç and D. Demirhan, Phys. Lett. A 181, 24 (1993); F. Büyükkılıç, D. Demirhan and A. Güleç, Phys. Lett. A 197, 209 (1995). 
[7] F. Büyükkılıç and D. Demirhan, Z. Phys. B 99, 137 (1995).

[8] U. Tirnaklı, C. Tsallis and M.L. Lyra, Phys. Rev. E 65, 036207 (2002).

[9] U. Tirnakll, Phys. Rev. E 66, 066212 (2002);

[10] A.K. Rajagopal, Phys. Rev. Lett. 74, 1048(1995).

[11] S. Abe and G.B. Bağcı, Phys. Rev. E 71, 016139 (2005).

[12] G.B. Bağc1, A. Arda and R. Sever, Int. J. Mod. Phys. B 20, 2085 (2006).

[13] C. Tsallis, J. Stat. Phys. 52, 479 (1988).

[14] E. Curando and C. Tsallis, J. Phys. A 24, L69 (1991); Corrigenda: 24, 3187 (1991) and 25, 1019 (1992).

[15] T. Haliloğlu, I. Bahar and B. Erman, Phys.Rev.Lett. 79,3090 (1997).

[16] E. K. Lenzi, R. S. Mendes, L. R. da Silva and L. C. Malacarne, Phys. Lett. A 44, 289 (2001).

[17] W. Greiner, L. Neise and H. Stöker, Thermodynamics and Statistical Mechanics, SpringerVerlag,(1995).

[18] G.B. Arfken and H.J. Weber, Mathematical Methods for Physicist, Academic Press, (2001).

[19] U. G. Wagner et al., J. Mol. Biol. 247, 326 (1995). 